\title{
Conceptual understanding and quantity inferences: a new framework for examining consumer understanding of food energy
}

\author{
Dawn Liu* and Marie Juanchich \\ Department of Psychology, University of Essex, Wivenhoe Park, Colchester, Essex, CO4 3SQ, UK
}

Submitted 12 January 2018: Final revision received 8 June 2018: Accepted 13 July 2018: First published online 22 August 2018

\begin{abstract}
Objective: The study examined two components of consumer understanding of food energy information: understanding the concept of energy and its quantity. Using this new framework, we investigated whether activity-equivalent labels facilitated interpretations of food energy compared with calorie labels and whether an image format would strengthen this facilitative effect compared with text.

Design: We assessed the effect of energy representation and format in a 2 (activity $v$. calories) $\times 2$ (image $v$. text) between-subjects design. Conceptual understanding of energy was measured in terms of level of understanding and personal engagement. Quantitative understanding was measured in terms of participants' estimations of a food's contribution to their recommended daily intake and perceptions of energy values as precise or single-bound interval estimates.

Setting: The experiment was conducted online through Qualtrics.

Subjects: Eight hundred and twelve participants (55\% female, age range 18-74 years) were recruited through a national survey panel in the UK.

Results: Participants were twice more likely to have a stronger conceptual understanding of energy and four times more likely to personally engage with activity labels $v$. calorie labels. Participants did not differ across labels in their estimations of energy quantities; however, they inferred quantities to mean exactly the stated number of calories, but at least the stated activity duration. There were no added benefits in presenting an image over the text format.

Conclusions: Activity labels can facilitate conceptual understanding of energy, but may be subject to quantitative misinterpretations. Nutrition communication should consider what people infer from quantities represented on labels.
\end{abstract}

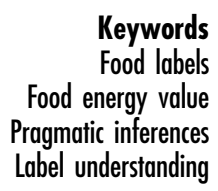

Keywords

Food energy value Pragmatic inferences Label understanding
In the UK, $60 \%$ of adults are overweight or obese, a condition associated with increased risk of chronic health conditions such as diabetes and heart disease ${ }^{(1)}$. With the prevalence of obesity expected to reach $70 \%$ in the next 20 years $^{(1)}$, it is important to develop solutions to counter this rising trend. The intake of energy-dense foods is one risk factor for obesity ${ }^{(2)}$, as energy intake exceeding energy expenditure leads to weight gain $^{(3)}$. Therefore measures to curb obesity include interventions to improve people's understanding of the amount of energy that is provided by food ${ }^{(4)}$. Energy is consistently included as key information in nutrition labelling in Europe ${ }^{(5)}$, highlighting that it should be a key consideration in making healthy food choices.

Studies suggest that providing information on energy content facilitates the making of healthy food choices ${ }^{(6)}$. This information is readily available as 'calories' (i.e. kilocalories; $1 \mathrm{kcal}=4.184 \mathrm{~kJ}$ ) on most food labels internationally ${ }^{(7)}$ and relied upon by consumers when considering nutrition ${ }^{(8)}$.
However, there is evidence that consumers often misunderstand energy information and do not in fact understand the meaning of a calorie or its contribution to their energy needs ${ }^{(9,10)}$. Further, calorie labelling can take different forms; for instance, physical activity labels that relate the energy concept to how it is used in daily activity, or percentage daily intake labels that place the energy quantity in the context of a daily dietary recommendation. There is mixed evidence about which type of calorie labelling is more effective than others ${ }^{(1-14)}$. This suggests that consumers' understanding of a label may have conceptual as well as quantitative components, but research has yet to combine the two types of understanding. The definition of food energy understanding and the measures used to capture this are varied, with each study offering information about a facet of the concept (e.g. Grunert and Wills ${ }^{(8)}$, Watson et al. $\left.{ }^{(15)}\right)$. The focus of the present paper is to integrate the different ways consumers may derive understanding about food energy information. 


\section{A new energy interpretation framework}

The new framework proposed herein addresses the multifaceted processes involved in understanding food energy information. Ideally, a consumer's understanding should match the meaning a communicator intended to provide ${ }^{(8)}$. Consumers thus need to interpret the term 'calories' as representing energy provided by the food, as well as the quantity provided ${ }^{(16)}$. The concept of energy and its quantity have been separately investigated ${ }^{(8,15,17)}$, but they have yet to be systematically examined in a unified framework. To provide a more holistic approach to the understanding of food energy labels, we developed an interpretation framework centred on these two pillars (see Fig. 1). Each pillar of interpretation (qualitative/conceptual and quantitative) was tapped into with two operational variables.

\section{Conceptual understanding of food energy}

To fully understand what food energy is, people must realise that it is fuel for the body and concretise this concept by placing the information in the context of their own lifestyle ${ }^{(15)}$. Both these two ways in which food energy can be conceptually understood affect people's use of the information. Being able to define what energy means allows consumers to understand its contribution to their health (and in particular, how excess energy intake leads to obesity $\left.{ }^{(15,16)}\right)$. Further, associating this concept to themselves should create personal relevance that makes the message to reduce consumption more persuasive ${ }^{(18)}$.

\section{Quantitative understanding of food energy}

There are two ways people can derive meaning about energy quantities, which contribute to how effectively they can use energy labels to make healthy choices. First, people can perceive quantities in terms of an estimation of a food's contribution to a daily recommended total. This gives people a context in which to interpret otherwise meaningless numbers ${ }^{(19)}$ and allows them to ascertain whether they are in fact eating too much. Second, people can identify the position of the given quantity within a distribution (e.g. is it a minimal, maximal, exact or rough estimate?). This modifies the meaning people take away from a given estimate: 'at least 100 calories' presents a very different message from 'up to 100 calories'.

To date, research on people's understanding of the quantities indicated on food labels has focused on the first way: their ability to identify exactly how much energy one is consuming (e.g. as part of a daily recommended intake ${ }^{(20)}$ ). However, the literature on pragmatics (i.e. the practical meaning people derive from information) suggests that people do not process quantities so precisely; rather, they infer the position of a numerical estimate based on conversational conventions ${ }^{(21)}$. In ordinary communication contexts, quantities are often used to refer to the extremes of a distribution, particularly when prefaced by modals such as 'can' and 'will'(22). In general, lower-bound estimates seem to be the default ${ }^{(23,24)}$, with people inferring that quantities are minimal amounts to be expected (e.g. 'two of the cereals are unhealthy' is taken to mean 'at least two of the cereals are unhealthy, ${ }^{,(25)}$; for a review, see Breheny ${ }^{(26)}$ ). However, when people expect the speaker to dislike an option, people infer that quantities refer to a maximal amount (e.g. 'you only get $5 \mathrm{~g}$ of fibre' would be inferred to mean 'up to $\left.5 g^{\prime}\right)^{(24)}$. These sorts of pragmatic inferences about energy quantities may affect people's understanding. However, it is a matter that has yet to be investigated.

\section{Identifying elements that facilitate food label understanding}

The interpretation framework presented incorporates aspects of energy label understanding that were previously independently investigated and adds new facets to it. This

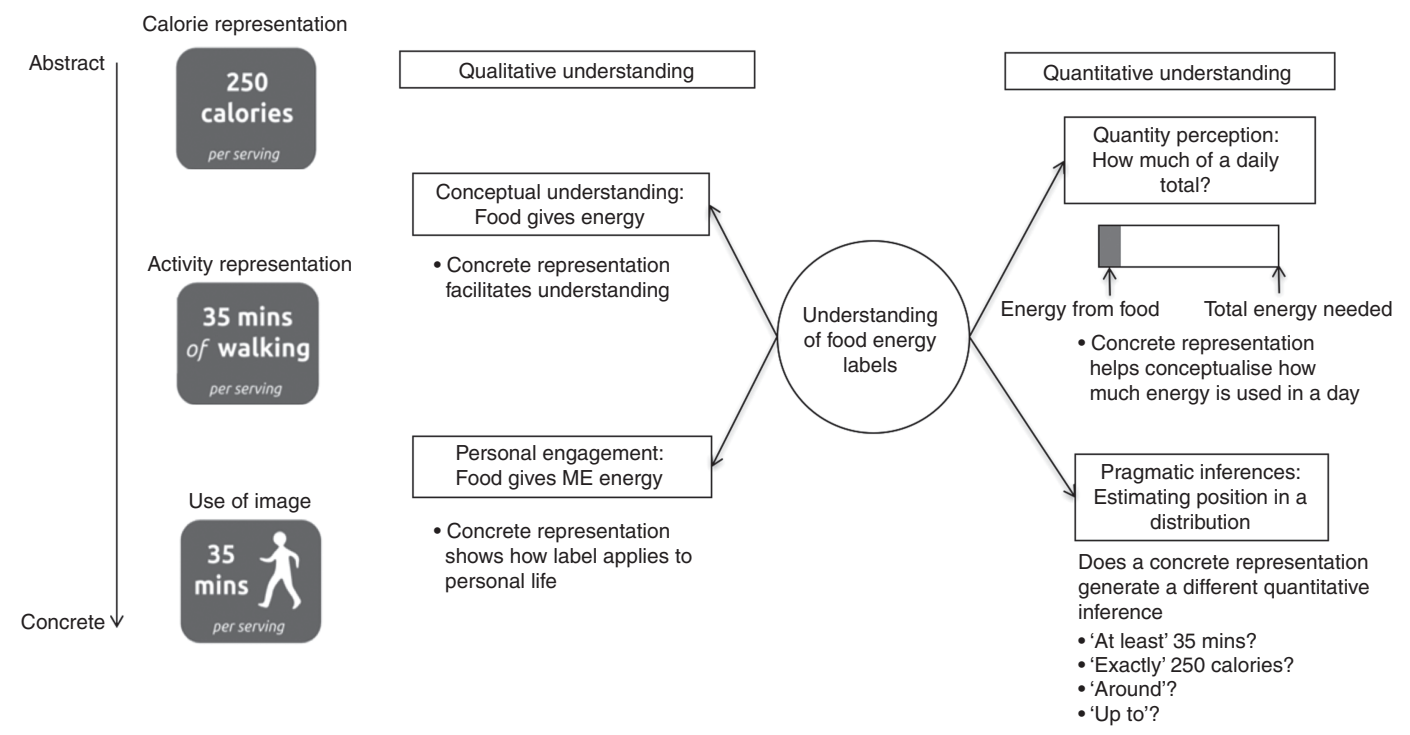

Fig. 1 A framework for energy interpretation 
integrative approach has an additional advantage in allowing us to assess more systematically the impact of specific variations in label types. One such variation in food energy labelling is a proposed 'physical activity label' (e.g. Royal Society for Public Health ${ }^{(27)}$ ), which presents food energy in terms of the activity time required to burn off the food.

It has been suggested that because physical activity time is more concrete, representing energy with these units makes it more easily understood than using 'calories, (27). In line with this hypothesis, presenting abstract concepts in concrete terms facilitates understanding of the concepts ${ }^{(28)}$. In addition, depicting energy in terms of physical activity may be more effective in prompting people to consider how the label applies to their own lifestyle and $\operatorname{diet}^{(29)}$. Conversely, people are less likely to relate the calorie content of food to themselves ${ }^{(29)}$. Representing energy in terms of activity time instead of calories is therefore posited to evoke a more accurate and personalised conceptual understanding of food energy ${ }^{(29)}$.

How would a label's energy representation affect the type of inferences made? We could expect that people would make the default inference that the energy quantity is a minimum to be expected whatever its representation or format ${ }^{(30)}$. Alternatively, people might see calories as a warning but activity time as an encouragement for action, resulting in differing perceptions of two estimates of the same energy value ${ }^{(24)}$ : a lower-bound interpretation of activity (e.g. 'the food requires at least 35 min of walking') infers a more energy-dense product than an upper-bound interpretation of calories (e.g. 'the food provides up to 250 calories'). Inferences about quantity estimates may therefore be informative about how people view activity and calorie labels respectively: as cautionary or encouraging.

A further variation introduced by physical activity labels is the format of presentation used, whereby calorie labels present information in text-only form (e.g. using the word 'calories') but activity labels convey conceptual information using an image to depict physical activity ${ }^{(10,27)}$. While past research comparing food labels that vary along more than one dimension (e.g. one label that presents fewer nutrients with verbal information $v$. another with more nutrients and numerical information ${ }^{(31)}$ ) allows conclusions to be drawn about how different labels affect food choice, the lack of systematicity limits our identification of what factors facilitate understanding. We overcame this limitation by manipulating orthogonally two aspects of a label. Given that images activate conceptual understanding better than words ${ }^{(32)}$, and that labels with images such as graphic displays or summary symbols are more easily understood by consumers than text- or number-only labels $^{(31)}$, we expected an image to reinforce conceptual understanding of food energy.

In the present work, we investigated experimentally whether representing food energy as activity time facilitated conceptual and quantitative understanding of energy compared with the calorie representation. We further tested whether the label's depiction as an image reinforced this facilitative effect. We hypothesised that consumers would understand activity and image labels better than calorie and text ones, and that activity times would be perceived as minimal quantities while calories would be perceived as maximal quantities. The materials and data for our experiments are available on the Open Science Framework (OSF) at the link http://www.osf.io/d2ugf/.

\section{Methods}

\section{Pilot study}

The materials and design were first tested in an online pilot study on ninety-six participants obtained through snowball sampling. This study enabled us to gauge the type of responses participants might provide to open questions regarding what an energy label meant and develop a coding framework to better capture participants' levels of understanding. In addition, it provided preliminary data that suggested participants would differ in their quantitative interpretation of energy estimates as minimal, maximal, exact or approximate quantities. A detailed report of this pilot study is available as supplementary materials on the OSF (https://www.osf.io/d2ugf/).

\section{Participants}

Using quota sampling to determine demographic parameters that would closely represent the UK population, we recruited 835 participants from a survey panel company. They completed the survey at the end of an unrelated questionnaire on how participants felt about seeing clusters of holes. After excluding incomplete data and careless responses (as specified in our preregistered experimental protocol, available on the OSF), we had 812 respondents (55\% female, age range 18-74 years). Participants' mean BMI $(27.72$ (SD 7.62) kg/m²) indicated an overweight tendency. Participants had slightly favourable attitudes towards healthy eating ${ }^{(33)}$ (mean 4.74 (SD 1.09)). Fifty-one per cent reported using nutritional labels. Full demographic characteristics for the sample are reported in Table 1.

\section{Design and procedure}

The experiment was conducted online using Qualtrics. Each participant was randomly assigned to one of four experimental conditions in a 2 (energy representation: calories or activity time) $\times 2$ (format: text or image) between-subjects design. Participants completed three tasks presented in a randomised order to each participant, followed by control and demographic questions.

\section{Conceptual understanding and engagement task}

To measure energy meaning, participants viewed a food label (see the top panel of Fig. 2) and gave open-ended responses about what the label meant. This qualitative 
answer was used to derive participants' level of conceptual understanding and engagement with the label. The coding scheme is presented in Table 2 .

\section{Quantity perception task}

To measure quantity perception, participants viewed the label attached to two foods (milk and chocolate, presented

Table 1 Sociodemographic characteristics of the experimental sample of 812 participants ( $55 \%$ female, age range $18-74$ years) recruited through a national survey panel in the UK

\begin{tabular}{lrr}
\hline & $n$ & $\%$ \\
\hline Age range (years) & & \\
18-24 & 51 & $6 \cdot 3$ \\
$25-34$ & 138 & $17 \cdot 1$ \\
$35-44$ & 168 & $20 \cdot 8$ \\
$45-54$ & 169 & $21 \cdot 0$ \\
$55-64$ & 137 & $17 \cdot 0$ \\
$65-74$ & 143 & $17 \cdot 7$ \\
Ethnicity & & \\
White/Caucasian & 712 & $88 \cdot 0$ \\
Asian & 49 & $6 \cdot 1$ \\
Black & 23 & $2 \cdot 8$ \\
Mixed & 11 & $1 \cdot 4$ \\
Other & 14 & $1 \cdot 7$ \\
Employment status & & \\
Full-time & 331 & $40 \cdot 8$ \\
Part-time & 127 & $15 \cdot 7$ \\
Self-employed & 55 & $6 \cdot 8$ \\
Student & 22 & $2 \cdot 7$ \\
Unemployed & 108 & $13 \cdot 3$ \\
Retired & 168 & $20 \cdot 7$ \\
Highest education level & & \\
High school or equivalent & 344 & $42 \cdot 4$ \\
Degree or higher & 290 & $35 \cdot 7$ \\
Apprenticeship & 38 & $4 \cdot 7$ \\
Other qualifications & 105 & $4 \cdot 3$ \\
No qualifications & 35 & \\
& &
\end{tabular}

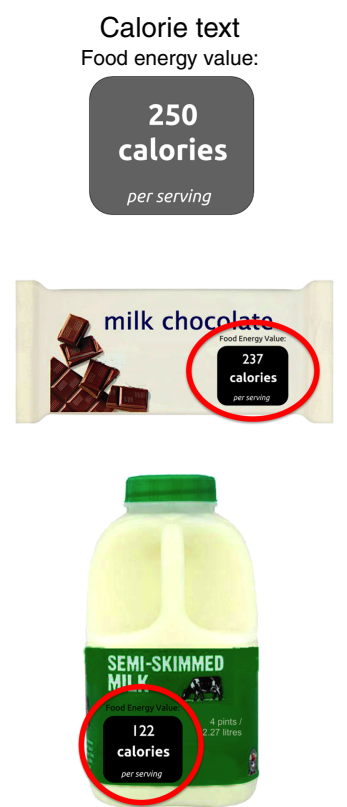

individually; see Fig. 2) and estimated on a sliding scale (anchor points: 0-100\%) what percentage of their daily energy requirement the food provided.

\section{Pragmatic inference task}

To measure pragmatic inferences made, participants viewed the same foods with the label and were asked to pick the best choice to complete the following sentence (example for milk and within parentheses for chocolate):

Calorie condition: 'If I consumed one serving (bar) of milk (chocolate), I would have consumed 122 (237) calories of energy.'

Activity condition: 'If I consumed one serving (bar) of milk (chocolate), I would have to walk 18

(33) min to use up the energy.'

The choices to complete the sentence were 'at least', 'exactly', 'around' or 'up to'.

\section{Control and demographic questions}

Participants estimated how many calories they were required to consume in a day and how much walking time this amount would confer. This allowed us to control for participants' existing knowledge about recommended calorie amounts. On average, participants gave fairly accurate estimates of what their total calorie intake should be (mean 1787 (sD 1368) kcal/d), but underestimated the time needed to walk this amount off (mean 156 (SD 341) $\mathrm{min}$ ). To control for experience with food labels, participants also indicated on a 7-point Likert scale ( $1=$ 'strongly disagree, $7=$ 'strongly agree') whether they agreed with 
Table 2 Coding scheme for levels of understanding and engagement

\begin{tabular}{|c|c|}
\hline Description & Example(s) \\
\hline \multicolumn{2}{|l|}{ Level of understanding } \\
\hline $\begin{array}{l}\text { Weak understanding: response lacks appreciation that the label refers } \\
\text { to food energy }\end{array}$ & $\begin{array}{l}\text { 'An attempt to make you think it is ok to consume it.' } \\
\text { 'The product probably contains a large amount of } \\
\text { saturated fat.' }\end{array}$ \\
\hline $\begin{array}{l}\text { Moderate understanding: response recognises that the label provides quantitative } \\
\text { information in terms of calories or activity time, but does not relate this } \\
\text { information to energy }\end{array}$ & $\begin{array}{l}\text { 'Means that this serving deserves } 250 \text { calories in } \\
\text { average.' } \\
\text { 'Calories in this product worth } 35 \text { min of walking.' }\end{array}$ \\
\hline $\begin{array}{l}\text { Strong understanding: response shows that the label is talking about energy } \\
\text { derived from the food }\end{array}$ & $\begin{array}{l}\text { 'That it would provide you with enough energy for } \\
35 \text { min of being active.' } \\
\text { 'It means that the food you are eating will give } 250 \mathrm{kcal} \\
\text { of energy.' }\end{array}$ \\
\hline \multicolumn{2}{|l|}{ Level of engagement } \\
\hline $\begin{array}{l}\text { Reference to person absent } \\
\text { Reference to person present }\end{array}$ & $\begin{array}{l}\text { 'It has } 250 \text { calories per serving.' } \\
\text { 'You'll require } 35 \text { min of walking to burn off the calories } \\
\text { intake for this amount of serving.' }\end{array}$ \\
\hline
\end{tabular}

Note. Use of personal pronouns was coded as present only when they were applied with regard to the part of the description that actually explained the meaning of the label.

the sentence, 'I often use nutritional labels to determine the healthiness of food.'

\section{Label development}

As shown in the top row of Fig. 2, four food labels were digitally designed to vary systematically the two independent variables (energy representation and format). We included only the energy information on these labels so as not to introduce other extraneous factors (e.g. additional nutrients). Text labels were chosen to convey the calorie and walking time information while keeping a consistent overall design. The full word 'calories' presented in the text-only calorie label corresponds with depictions in existing label systems ${ }^{(34)}$. Graphic designs were based on proposed designs and stimuli that had been used in previous research for activity ${ }^{(10,27,29)}$ and calories ${ }^{(35)}$. For the quantity perception and pragmatic inference tasks, the values attached to foods (milk and chocolate) were calculated based on ballpark figures for the energy value of the food ${ }^{(36)}$ and the mean weight of someone living in the $\mathrm{UK}^{(37)}$. Figure 2 shows the four label designs, along with their presentation with the two foods used in the survey.

\section{Data coding and preparation}

\section{Coding scheme for energy meaning}

Participants' responses to the question of what the energy label meant provided an indication of both their conceptual understanding of energy and the level of personal engagement with the energy information. We developed two categories of codes (presented in Table 2) to measure two components of meaning suggested by our proposed energy interpretation framework.

Level of understanding. Level of understanding was coded according to how well participants understood the labels to indicate that energy from food could be used by the body (a theme highlighted in Watson et al.'s ${ }^{(15)}$ earlier work on consumer understanding of food energy). Based on the sample of responses provided in the pilot study, we derived three coding levels that corresponded to a weak, moderate or strong understanding of the energy information. The development of the coding scheme was first tested on the pilot data by two research assistants blind to the hypotheses, with disagreements resolved through discussion. The final coding scheme showed good interrater reliability on the pilot data $(\kappa=0.805, P<0.001)$ and was subsequently applied to the experimental data by a research assistant blind to the hypotheses, with the first author coding $10 \%$ of the cases to check reliability ( $\kappa=0.621, \quad P<0.001)$. Disagreements were resolved through discussion.

Level of engagement. Level of engagement was coded according to the use of first- and second-person pronouns (i.e. 'I', 'you' $v$. 'it') because first- and second-person pronouns generally indicate a higher level of interactivity and engagement than third-person pronouns ${ }^{(38)}$. This coding scheme was also tested on the pilot data before being applied to the experimental data. Inter-rater reliability on this criterion was good $(\kappa=0.843, P<0.001$ in the pilot data; $\kappa=0.902, P<0.001$ in the experimental data).

\section{Quantity meaning}

We measured the accuracy of participants' energy estimates in terms of how far off they were from the actual percentage of a daily total the given energy figure indicated. Although a guideline daily calorific requirement is 2000 calories $^{(39)}$, we based estimation accuracy on a range of 1500-2500 daily calories to accommodate for variation in people's calorific needs. For example, the 122-calorie milk would provide $5-8 \%$ of daily energy requirements. We measured errors in estimation as the absolute deviation from the respective upper or lower boundary for all estimates that fell outside this range. Estimation errors were averaged across milk and chocolate. 


\section{Analytical strategy}

Energy meaning

The conceptual understanding and personal engagement variables were entered as outcome variables in a logistic regression using energy representation and format as predictors and eating attitudes, BMI and frequency of label use as covariates. The models showed a good fit $\left(\chi_{(6)}^{2}=45.53\right.$, $P<0.001$, Nagelkerke $R^{2}=0.08$ (ordinal logistic regression); $\chi_{(5)}^{2}=53.53, P<0.001$, Nagelkerke $R^{2}=0.13$ (binary logistic regression)).

\section{Quantity meaning}

Estimation errors were analysed in an ANCOVA with energy representation and format as between-subject factors, and BMI, eating attitude, frequency of label use, age, gender and daily calorie intake estimates as covariates. The type of pragmatic inferences participants selected was analysed in a multinomial regression using rough estimates ('around') as the reference value, energy representation and format as predictors, and eating attitudes, BMI and frequency of label use as covariates (model fit: $\chi_{(15)}^{2}=113.34, P<0.001$, Nagelkerke $R^{2}=0 \cdot 09$; see Table 3 ).

\section{Results}

\section{Conceptual understanding of energy}

Level of understanding

Overall, only $20 \%$ of participants displayed a strong understanding of energy. Supporting our hypothesis, $29 \%$ of participants who viewed activity labels had such an understanding ( $v$. 11\% of those who viewed calorie labels). More participants also showed a strong understanding with image than text labels (22\% v. 17\%). Table 3 shows the parameter estimates obtained in the ordinal regression. Activity labels were about twice more likely than calorie labels to evoke stronger understanding $(P=0 \cdot 005)$. However, there was no indication that format (image $v$. text) affected participants' level of understanding for calorie or activity labels. Stronger understanding was also predicted by healthier eating attitudes $(P=0.001)$ and lower BMI $(P=0 \cdot 001)$, but not frequency of label use.

\section{Level of engagement}

Thirty-one per cent of all participants engaged with the labels. Those who saw activity labels engaged more than those who saw calorie labels ( $44 \% v .17 \%$ ). Participants also engaged more with text labels than image labels (33\% $v$. $28 \%$ ). The binary regression (see Table 3 ) showed that participants were four times more likely to use personrelated pronouns to describe the meaning of activity labels than calorie labels $(P<0 \cdot 001)$. However format again did not have a significant effect on engagement level. The covariates also did not predict different levels of engagement.

\section{Quantitative understanding of energy}

\section{Accuracy of quantity perception}

Overall, participants overestimated the contribution of the milk and chocolate to their daily recommended intake (mean $12 \cdot 14$ (SD 18.02) \%), and this was not affected by the energy representation of the label or its format (all $P>0 \cdot 40$ ). Table 4 reports in full the $P$ values and effect sizes obtained in the ANCOVA. Participants with higher BMI were more likely to overestimate the food's contribution towards their daily

Table 3 Parameter estimates from the ordinal, binary and multinomial logistic regression models for level of understanding, level of engagement and pragmatic inferences among the experimental sample of 812 participants ( $55 \%$ female, age range $18-74$ years) recruited through a national survey panel in the UK

\begin{tabular}{|c|c|c|c|c|c|c|c|c|}
\hline & \multicolumn{4}{|c|}{ Level of understanding (ordinal) } & \multicolumn{4}{|c|}{ Level of engagement (binary) } \\
\hline & $b$ & $x^{2}$ & $P$ & OR & $b$ & $x^{2}$ & $P$ & OR \\
\hline Energy representation (ref. $=$ calories) & 0.64 & 8.01 & 0.005 & 1.89 & 1.61 & 0.28 & $<0.001$ & $5 \cdot 01$ \\
\hline Format $($ ref. $=$ text $)$ & -0.08 & 0.12 & 0.731 & 0.93 & 0.06 & 0.32 & 0.840 & 1.07 \\
\hline Energy representation $\times$ format & 0.21 & 0.47 & 0.495 & 1.24 & -0.54 & 0.40 & $0 \cdot 170$ & 0.58 \\
\hline Eating attitudes & 0.28 & 11.89 & 0.001 & 1.33 & 0.16 & 0.10 & 0.101 & $1 \cdot 18$ \\
\hline $\mathrm{BMI}$ & -0.03 & $10 \cdot 14$ & 0.001 & 0.97 & -0.01 & 0.01 & 0.717 & 1.00 \\
\hline Frequency of label use & -0.06 & $1 \cdot 12$ & 0.289 & 0.95 & -0.04 & 0.07 & 0.527 & 0.96 \\
\hline
\end{tabular}

Pragmatic inferences (multinomial)

\begin{tabular}{|c|c|c|c|c|c|c|c|c|c|c|c|c|}
\hline & \multirow{2}{*}{\multicolumn{4}{|c|}{ Minimal estimate }} & & & & & & & & \\
\hline & & & & & \multicolumn{4}{|c|}{ Exact estimate } & \multicolumn{4}{|c|}{ Maximal estimate } \\
\hline & $b$ & $x^{2}$ & $P$ & OR & $b$ & $x^{2}$ & $P$ & OR & $b$ & $x^{2}$ & $P$ & OR \\
\hline Energy representation (ref. $=$ calories) & 0.73 & 25.98 & $<0.001$ & 2.08 & -0.68 & $20 \cdot 56$ & $<0.001$ & 0.51 & 0.01 & 0.002 & 0.965 & 1.01 \\
\hline Format (ref. $=$ text) & -0.19 & 1.76 & 0.185 & 0.83 & 0.03 & 0.05 & 0.824 & 1.03 & 0.33 & 1.72 & 0.190 & 1.39 \\
\hline Eating attitudes & -0.03 & 0.14 & 0.709 & 0.97 & 0.05 & 0.37 & 0.545 & 1.05 & -0.01 & 0.003 & 0.956 & 0.99 \\
\hline BMI & 0.02 & 5.05 & 0.025 & 1.02 & 0.03 & $6 \cdot 29$ & 0.012 & 1.03 & 0.03 & 3.77 & 0.052 & 1.03 \\
\hline Frequency of label use & 0.11 & 4.55 & 0.033 & $1 \cdot 11$ & 0.03 & 0.30 & 0.581 & 1.03 & -0.12 & 2.07 & 0.150 & 0.89 \\
\hline
\end{tabular}

ref., reference category.

Note. All Wald $x^{2}$ values were calculated with $\mathrm{df}=1$. 
Table 4 Effects of energy representation and format on errors in estimation of energy quantities (analysed in a between-subjects ANCOVA) by the experimental sample of 812 participants (55\% female, age range 18-74 years) recruited through a national survey panel in the UK

\begin{tabular}{lccc}
\hline & $F$ & $P$ & $\eta_{\mathrm{p}}^{2}$ \\
\hline Factor & & & \\
$\quad$ Energy representation & 0.21 & 0.649 & $<0.001$ \\
Format & 0.31 & 0.581 & $<0.001$ \\
$\quad$ Energy representation $\times$ format & 0.61 & 0.434 & 0.001 \\
Covariate & & & \\
Eating attitudes & 2.26 & 0.133 & 0.004 \\
BMl & 8.86 & 0.003 & 0.04 \\
Frequency of label use & 0.002 & 0.968 & $<0.001$ \\
Daily intake estimate & 25.77 & $<0.001$ & 0.04 \\
Age & 3.68 & 0.055 & 0.01 \\
Gender & 0.09 & 0.766 & $<0.001$ \\
\hline
\end{tabular}

Note. All $F$ values were calculated at $\mathrm{df}=1618$.

requirement $(P=0.003)$. Also, participants who recommended consuming fewer calories daily estimated foods as contributing more to their daily total $(P<0 \cdot 001)$. Therefore, how well participants could integrate energy information into their daily consumption was more influenced by their existing nutritional knowledge than by the features of the energy label.

\section{Pragmatic inferences about energy values}

Numbers on the label were inferred to be rough approximations ('around') about a third of the time. The multinomial regression (see Table 3 ) showed that participants perceived activity labels to be a minimal estimate twice more often as calorie labels, but inferred calorie labels to be exact estimates twice more often than activity labels (both $P<0 \cdot 001$ ). This pattern was consistent across image and text formats (see Fig. 3).

\section{Discussion}

We investigated people's understanding of food energy in a new framework that included two dimensions of understanding: understanding of the concept and of the quantity. Within this framework, we investigated how the representation of energy and its format of presentation affected people's understanding of food energy. As we predicted, participants showed a stronger understanding and greater personal engagement for activity than calorie labels. However, activity labels did not help participants better estimate how much a quantity of food contributed to a recommended daily energy intake. Delving further into participants' interpretation of quantities, we found that they inferred minimal estimates from activity labels and exact estimates from calorie labels, but format did not affect the type of inferences made.

\section{Do people understand the concept of food energy?}

Responses that spontaneously referred to energy, such as 'the food gives you enough energy to walk 35 min' and 'for every serving of the food the energy value to your
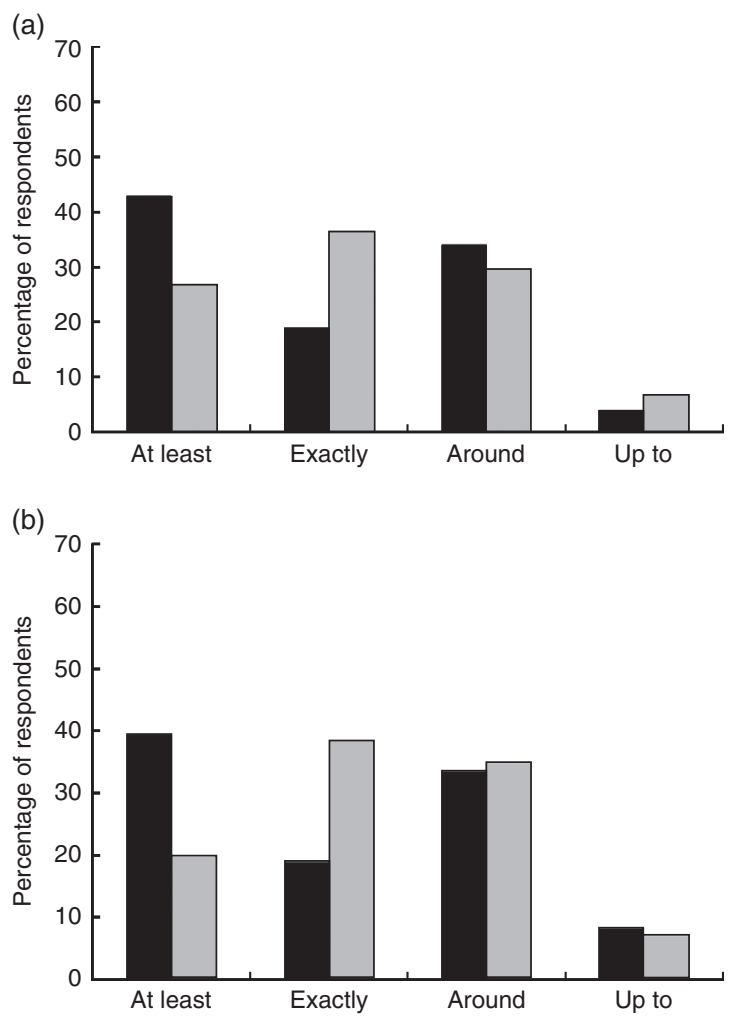

Fig. 3 Percentage of pragmatic inferences regarding energy label quantities (whether participants viewed food energy to be 'at least', 'exactly', 'around' or 'up to' the quantity given) by energy and format of the label among 812 participants (55\% female, age range 18-74 years) recruited through a national survey panel in the UK: (a) $\square$, activity text; $\square$, calorie text; (b) $\square$, activity image; $\square$, calorie image

body is 250 calories', reflected a strong understanding of what energy labels are communicating. Participants were more likely to report such understanding of food energy when shown activity labels as compared with calorie labels. Furthermore, based on activity labels, participants were up to four times more likely to apply this information to themselves. Our evidence therefore substantiates previous suggestions from focus group research that people's interpretations of activity labels are more accurate and 
personal than calorie labels ${ }^{(29)}$. However, we did not find further facilitating effects of image over text. While we posited from past research that images should improve conceptual understanding, it is possible 'activity time' is already clear enough in text format such that the additional benefit of using an image is minimal.

\section{How do people perceive energy quantities?}

Our interpretation framework included two elements of quantitative understanding. First was the ability to contextualise quantities as a proportion of a total, which has previously been relied upon as a measure of understanding (e.g. Grunert et al. ${ }^{(20)}$ ). Here, we found that activity labels did not provide a more accurate perception of the quantity of energy provided by food. Instead, how accurately participants were able to perceive a food's contribution to their daily energy requirements was determined more by their knowledge of daily calorie recommendations. The second was the pragmatic inferences people drew about energy quantities: whether people infer the given quantity to indicate an exact estimate, an approximate one, or one that specifies the lower or upper bound of a range. Past work has assessed understanding of quantities by the ability to perform accurate computations using the numbers (e.g. Malam et $a l .{ }^{(40)}$, Rothman et $\left.a l .{ }^{(41)}\right)$; however, this assumes that people believe the quantities to be exact. In our study, more than $65 \%$ of our participants did not consider the food label values to be exact estimates. This complements research from psycholinguistics that shows people apply communicative conventions when they interpret numerical quantities ${ }^{(23,24)}$. It may also indicate some awareness that energy labels are in fact variable estimates of the true energy value of the food ${ }^{(42,43)}$. However, if people interpret quantities to represent a range of possible values, this may contribute to their confusion in performing nutritional calculations ${ }^{(31)}$.

The effect of the energy representation on participants' inferences about quantities is a novel finding and one that merits further investigation. Participants were more likely to infer energy values to be minimal estimates ('at least') of activity time, but to be more often exact estimates of calories. For example, people believed they would have to walk at least 33 min to expend the energy in a chocolate bar, but they believed the chocolate had exactly 237 calories, although both referred to an equivalent amount of energy, which should be assumed to be equally vague. However, this did not result in a greater overestimation of how much activity-labelled food contributed more to their daily recommended total. It is thus uncertain whether participants were able to contextualise the food within their daily life despite seeing it as more calorific, or if they revised how much energy they thought they needed because they took the activity label as a sign that they required more energy in a day for their activities ${ }^{(15)}$.

\section{Practical applications}

The study showed that compared with calorie labels, activity labels were able to produce stronger and more personal conceptual understanding about energy. This helps to contextualise energy information, which enhances usability of the information in selecting healthier foods ${ }^{(44,45)}$. This suggests that activity labels are a promising intervention to improve food choices. However, the lack of a significant effect for the image format prompts the question of whether it is worthwhile to introduce images on food energy labels, especially since redesigning labels to include images can be twice as costly to manufacturers than implementing text changes ${ }^{(46)}$.

We did not find activity labels to be more beneficial than calorie ones in facilitating quantitative understanding. However, the change in energy representation affected the type of inferences people drew about energy quantities, with activity labels more often taken to be the minimal time taken to use up food energy, as opposed to calorie labels, which were taken as an exact energy quantity. From a communicative standpoint, if activity labels are to be used, it may be prudent to educate people about the distributions of the quantities so as to avoid misunderstandings about the quantity values. For instance, if people assume activity labels to be minimum walk times, they would be misinterpreting labels that intend to communicate the average time needed.

\section{Limitations and future research}

One limitation of the work is that with our focus on label understanding, we did not extend our measures to food choice. We focused on understanding because it is a precursor to label use ${ }^{(8)}$ and thus it is important to first ascertain if people are indeed deriving meaning from information as it is intended. However, future research could consider whether different inferences about the quantities of energy provided are relied upon in food decision making.

A further question not yet answered by our research is also how an 'activity time' representation of energy might work when the representation of quantities is not absolute, such as on percentage daily value labels. We did not find an effect of energy representation on quantity perception for an absolute energy quantity; however, if the recommended amount is standardised for participants, it may be that the tendency to infer activity times to be minimum quantities would then carry over to their proportion estimates. On the other hand, the use of a standardised quantity could interfere with the facilitative effect of activity labels in personalising information, since the stronger conceptual understanding produced might then prompt the realisation that the quantity indicated is not based on one's specific caloric requirements.

The landscape of nutrition labelling is highly complex, with many ways to present nutritional concepts and 
quantities. The challenge for public policy is to determine how to convey this information in an accurate and accessible way. Our study adds to the existing body of literature on the interpretation of information on food labels and offers greater insight as to how label design can be manipulated to fit the way people interpret the concept of energy and its quantity. In particular, communications about nutritional values need to consider not only how easy a conceptual representation or format is to understand, but also what people might infer from the manufacturer's choice of that representation or format.

\section{Acknowledgements}

Acknowledgements: The authors thank Guyan Sloane, Anca Pop, Amalia Cerb, Jessica Ng and Erica Hall for their contributions to the coding. Financial support: This research received no specific grant from any funding agency in the public, commercial or not-for-profit sectors. Conflict of interest: None: Authorship: The research question was formulated by D.L. and the design was a collaboration between D.L. and M.J. Data collection and analysis and the writing of the article was done by D.L., with help and input from M.J. Ethics of human subject participation: This study was conducted according to the guidelines laid down in the Declaration of Helsinki and all procedures involving human subjects were approved by the Research Ethics Committee of the University of Essex. Written informed consent was obtained from all participants.

\section{References}

1. Public Health England (2015) Making the Case for Tackling Obesity - Why Invest? London: Department of Health.

2. Swinburn BA, Caterson I, Seidell JC et al. (2004) Diet, nutrition and the prevention of excess weight gain and obesity. Public Health Nutr 7, 123-146.

3. Jequier E \& Tappy L (1999) Regulation of body weight in humans. Physiol Rev 79, 451-480.

4. Storcksdieck genannt Bonsmann S \& Wills JM (2012) Nutrition labeling to prevent obesity: reviewing the evidence from Europe. Curr Obes Res 1, 134-140.

5. Storcksdieck genannt Bonsmann S, Férnández Celemín L, Larrañaga A et al. (2010) Penetration of nutrition information on food labels across the EU-27 plus Turkey. Eur J Clin Nutr 64, 1379-1385.

6. Littlewood JA, Lourenço S, Iversen CL et al. (2016) Menu labelling is effective in reducing energy ordered and consumed: a systematic review and meta-analysis of recent studies. Public Health Nutr 19, 2106-2121.

7. Wartella EA, Lichtenstein AH, Yaktine A et al. (2011) Frontof-Package Nutrition Rating Systems and Symbols: Promoting Healthier Choices. Washington, DC: National Academy of Sciences.

8. Grunert KG \& Wills JM (2007) A review of European research on consumer response to nutrition information on food labels. J Public Health 15, 385-399.

9. Cowburn G \& Stockley L (2005) Consumer understanding and use of nutrition labelling: a systematic review. Public Health Nutr 8, 21-28.
10. Van Kleef E, Van Trijp H, Paeps F et al. (2008) Consumer preferences for front-of-pack calories labelling. Public Health Nutr 11, 203-213.

11. Masic U, Christiansen P \& Boyland EJ (2017) The influence of calorie and physical activity labelling on snack and beverage choices. Appetite 112, 52-58.

12. Fitch RC, Harnack LJ, Neumark-Sztainer DR et al. (2009) Providing calorie information on fast-food restaurant menu boards: consumer views. Am J Health Promot 24, 129-132.

13. Morley B, Scully M, Martin J et al. (2013) What types of nutrition menu labelling lead consumers to select less energydense fast food? An experimental study. Appetite 67, 8-15.

14. Ellison B, Lusk JL \& Davis D (2013) Looking at the label and beyond: the effects of calorie labels, health consciousness, and demographics on caloric intake in restaurants. Int $J$ Behav Nutr Phys Act 10, 21.

15. Watson WL, Chapman K, King L et al. (2013) How well do Australian shoppers understand energy terms on food labels? Public Health Nutr 16, 409-417.

16. British Nutrition Foundation (2018) What is energy? http:// www.nutrition.org.uk/healthyliving/basics/what-is-energy. html (accessed April 2018).

17. Vanderlee L, Goodman S, Sae Yang W et al. (2012) Consumer understanding of calorie amounts and serving size: implications for nutritional labelling. Can J Public Health 103, e327-e331.

18. Petty RE \& Cacioppo JT (1986) The elaboration likelihood model of persuasion. Adv Exp Soc Psychol 19, 123-205.

19. Auchincloss AH, Young C, Davis AL et al. (2013) Barriers and facilitators of consumer use of nutrition labels at sitdown restaurant chains. Public Health Nutr 16, 2138-2145.

20. Grunert KG, Wills JM \& Fernandez-Celemin L (2010) Nutrition knowledge, and use and understanding of nutrition information on food labels among consumers in the UK. Appetite 55, 177-189.

21. Grundy P (2014) Pragmatic inference. In The Encyclopedia of Applied Linguistics [CA Chapelle, editor]. Chichester: John Wiley \& Sons. https://doi.org/10.1002/9781405198431. wbeal1449.

22. Teigen KH, Juanchich M \& Filkukova P (2014) Verbal probabilities: an alternative approach. QJ Exp Psychol 67, 124-146.

23. Mandel DR (2015) Communicating numeric quantities in context: implications for decision science and rationality claims. Front Psychol 6, 537.

24. Halberg A-M \& Teigen KH (2009) Framing of imprecise quantities: When are lower interval bounds preferred to upper bounds? J Behav Decis Mak 22, 490-509.

25. Horn LR (1984) A new taxonomy for pragmatic inference: Q-based and R-based implicature. In Meaning, Form and Use in Context, pp. 11-42 [D Schiffrin, editor]. Washington, DC: Georgetown University Press.

26. Breheny R (2008) A new look at the semantics and pragmatics of numerically quantified noun phrases. J Semantics 25, 93-139.

27. Royal Society for Public Health UK (2016) Introducing 'Activity Equivalent' Calorie Labelling to Tackle Obesity. London: RSPH.

28. Barrio PJ, Goldstein DG \& Hofman JM (2016) Improving comprehension of numbers in the news. In Proceedings of the 2016 CHI Conference on Human Factors in Computing Systems, pp. 2729-2739. New York: Association for Computing Machinery.

29. Swartz JJ, Dowray S, Braxton D et al. (2013) Simplifying healthful choices: a qualitative study of a physical activity based nutrition label format. Nutr $J \mathbf{1 2}, 72$.

30. Mandel DR (2014) Do framing effects reveal irrational choice? J Exp Psychol Gen 143, 1185-1198.

31. Campos S, Doxey J \& Hammond D (2011) Nutrition labels on pre-packaged foods: a systematic review. Public Health Nutr 14, 1496-1506. 
32. McBride DM \& Dosher A (2002) A comparison of conscious and automatic memory processes for picture and word stimuli: a process dissociation analysis. Conscious $\operatorname{Cog} n$ 11, 423-460.

33. Steptoe A, Pollard TM \& Wardle J (1995) Development of a measure of the motives underlying the selection of food: the food choice questionnaire. Appetite 25, 267-284.

34. Becker MW, Bello NM, Sundar RP et al. (2015) Front of pack labels enhance attention to nutrition information in novel and commercial brands. Food Policy 56, 76-86.

35. Kiefer HC (1996) Process for analyzing nutritional content of a composition of food to produce a calculated printed representation. US Patent no. 5558742.

36. Department of Health UK (2017) Calorie checker. http:// www.nhs.uk/Tools/Pages/Calorie-checker.aspx (accessed November 2017).

37. British Broadcasting Corporation UK (2010) Statistics reveal Britain's 'Mr and Mrs Average'. http://www.bbc.co.uk/ news/uk-11534042 (accessed April 2016).

38. Yeo J-Y \& Ting S-H (2014) Personal pronouns for student engagement in arts and science lecture introductions. English for Specific Purposes 34, 26-37.

39. Department of Health UK (2016) Technical Guidance on Nutrition Labelling. London: Department of Health.
40. Malam S, Clegg S, Kirwan S et al. (2009) Comprehension and Use of UK Nutrition Signpost Labelling Scheme. London: BMRB Social Research.

41. Rothman RL, Housam R, Weiss H et al. (2006) Patient understanding of food labels: the role of literacy and numeracy. Am J Prev Med 31, 391-398.

42. Jumpertz R, Venti CA, Le DS et al. (2013) Food label accuracy of common snack foods. Obesity (Silver Spring) 21, 164-169.

43. Urban LE, Dallal GE, Robinson LM et al. (2010) The accuracy of stated energy contents of reduced-energy, commercially prepared foods. J Am Diet Assoc 110, 116-123.

44. Bleich SN \& Pollack KM (2010) The publics' understanding of daily caloric recommendations and their perceptions of calorie posting in chain restaurants. BMC Public Health 10, 121 .

45. Sinclair SE, Cooper M \& Mansfield ED (2014) The influence of menu labeling on calories selected or consumed: a systematic review and meta-analysis. J Acad Nutr Diet 114, 1375-1388.e15.

46. Balazs-Horvath M, Craddock N \& Morgan A (2010) Developing a Framework for Assessing the Costs of Labelling Changes in the UK. Chipping Camden: Campden BRI. 